\title{
Stereotypic Morphology of Glutamatergic Synapses on Identified Muscle Cells of Drosophila Larvae
}

\author{
Jørgen Johansen, Marnie E. Halpern, Kristen M. Johansen, and Haig Keshishian \\ Department of Biology, Yale University, New Haven, Connecticut 06511
}

The distribution and morphology of glutamatergic synapses on Drosophila bodywall muscle fibers were examined at the single-synapse level using immunocytochemistry and electrophysiology. We find that glutamate-immunoreactive motor endings innervate the entire larval bodywall musculature, with each muscle fiber receiving at least one glutamatergic ending. The innervation is initiated at stereotyped locations on each muscle fiber from where moderately branched varicose nerve processes project over the internally facing muscle surface. Individual muscle fibers have distinct stereotypic patterns of nerve endings that occupy characteristic regions on the cell surface. The muscle-specific branching pattern of motor endings is reiterated by segmentally homologous fibers. Two morphological types of innervating nerve processes can be distinguished by their bouton size distributions: (1) Type I processes, which have localized branching and a broad size distribution of relatively large varicosities ranging up to $8 \mu \mathrm{m}$ (mean diameter, $3.1 \pm 1.6$ $\mu \mathrm{m} ; \pm \mathrm{SD}, n=521$ ), and (2) thinner Type II processes, which have a narrower distribution of small varicosities with a mean diameter of only $1.4 \pm 0.6 \mu \mathrm{m}( \pm S D, n=214)$. Immunoelectron microscopy with peroxidase-labeled second antibody demonstrates that the varicosities are surrounded by a subsynaptic reticulum, that they contain immunoreactive vesicles of about 30-50 nm, and thus probably represent synaptic release sites. By iontophoretic application of glutamate we mapped the responsive sites on the muscle surface and found an excellent correspondence between transmitter sensitivity and the patterns of endings as described by immunocytochemistry. In contrast to our finding of numerous glutamate iontophoresis-sensitive sites, we did not detect any aspartate-responsive muscles. These data provide strong new evidence for glutamate being an endogenous transmitter at the Drosophila larval neuromuscular junction.

The bodywall of Drosophila larvae is a particularly favorable preparation for the analysis of motor innervation, as each hemisegment is composed of only 31 or fewer discrete unicellular muscle fibers. The muscle cells are organized in a stereotyped and segmentally repeated pattern (Hertweck, 1931; Crossley,

\footnotetext{
Received Apr. 22, 1988; revised June 30, 1988; accepted July 5, 1988.

We are grateful to Drs. P. Petrusz and C. Toomim for generously providing us with the glutamate antiserum. We also wish to thank Drs. J. Carlson, M. Constantine-Paton, and R. Wyman for helpful comments on the manuscript. This work was supported by grants from NSF, The March of Dimes, and the McKnight Foundation to H.K., a NIMH Training Grant (J.J.), a Canadian NSERC postgraduate scholarship to M.E.H., and a NSF graduate scholarship to K.M.J.

Correspondence should be addressed to Jorgen Johansen, Department of Biology, Yale University, KBT, Box 6666, New Haven, CT 06511.

Copyright (C) 1989 Society for Neuroscience $0270-6474 / 89 / 020710-16 \$ 02.00 / 0$
}

1978; Campos-Ortega and Hartenstein, 1985; Anderson et al., 1988). Electrophysiological and anatomical evidence suggests that muscle fibers in dipteran larvae are at least dually innervated by a "slow" and a "fast" excitatory axon (Jan and Jan, 1976a; Hardie, 1976; Irving and Miller, 1980a, b), which can be distinguished by their different thresholds to excitation and by their relative conduction velocity. For Drosophila larvae electrophysiological experiments indicate that the amino acid glutamate is either a neurotransmitter or a closely related agonist at the neuromuscular junction (Jan and Jan, 1976b). This is based on the observation that glutamate applied by iontophoresis can elicit depolarizations of the muscle fiber that have the same reversal potential as nerve-evoked EPSPs. Also, millimolar concentrations of bath-applied glutamate desensitize the EPSPs. However, it has not been possible to demonstrate the compartmentalization of glutamate to the presynaptic motor endings. In this study we have applied an immunocytochemical approach to this problem and examine in detail the morphology and distribution of glutamatergic synapses using a glutamatcspecific antibody at the level of both light and electron microscopy. Our experiments provide the first comprehensive visualization and description of the anatomy of glutamatergic neuromuscular junctions in Drosophila larvae. An unexpected finding from this analysis is that each muscle fiber has a distinctive and stereotyped pattern of innervation, which is reiterated by segmentally homologous muscle fibers. An interesting implication from this observation is that each muscle fiber may possess regional cell-surface recognition factors that are necessary for the different and specific innervation patterns to be established. Furthermore, our results present strong immunocytochemical evidence for glutamate being present in presynaptic specializations providing an important addition to the list of criteria necessary for a rigorous identification and demonstration of glutamate as an endogenous transmitter at the Drosophila neuromuscular junction.

We also reexamined the role of aspartate as a neuromuscular transmitter in dipteran larvae by means of intracellular recording and voltage-clamp experiments. Irving and Miller (1980b) reported that, in addition to glutamate, the muscle fibers of the house fly Musca are responsive to aspartate. These workers suggested that glutamate was the transmitter of the "fast" axon, whereas aspartate was the transmitter at the "slow" junction. However, in an attempt to repeat their work, Robinson (1981) was unable to detect a physiological effect of aspartate in Lucilia larvae. In our study, electrophysiological experiments conducted in both Drosophila and Calliphora larvae are consistent with the findings of Robinson (1981) and suggest that aspartate is not likely to be an endogenous transmitter at the larval neuromuscular junction in these insects, but rather that glutamate is a transmitter at both the fast and slow junction. Some of the 
material in this study has been reported in abstract form (Johansen et al., 1987).

\section{Materials and Methods \\ Preparations and solutions}

Stocks. Wild-type Canton S Drosophila melanogaster stocks were maintained under standard laboratory conditions at $21-23^{\circ} \mathrm{C}$ in uncrowded bottles. Crawling third instar larvae were used exclusively for the anatomical and physiological studies. Larvae of the blowfly Calliphora erythrocephala, a gift from Dr. T. Goldsmith, Yale University, were maintained on beef liver.

Saline. Osmotically balanced insect saline solutions consisted of 140 тм NaCl, 5 мм KCl, 5 mм $\mathrm{CaCl}_{2}, 4$ mм $\mathrm{NaHCO}_{3}, 1 \mathrm{~mm} \mathrm{MgCl}_{2}, 5$ mM $N$-Tris(hydroxymethyl) methyl-2-aminoethanesulfonic acid (TES), $5 \mathrm{~mm}$ trehalose, and $100 \mathrm{~mm}$ sucrose at $\mathrm{pH} 7.2$. For the immunocytochemistry and some of the physiology experiments, a $0 \mathrm{~mm} \mathrm{Ca}, 6 \mathrm{~mm}$ $\mathrm{Mg}$ version of this saline was used to suppress synaptic activity. For desensitization experiments the sodium salt of glutamate (glu) or aspartate (asp) (1-10 mm) was dissolved directly in the insect saline and the $\mathrm{pH}$ adjusted to 7.2 with $I \mathrm{~N} \mathrm{NaOH}$.

Reagents. Na glutamate, $\mathrm{Na}$ aspartate, BSA, normal goat serum (NGS), and GABA were obtained from Sigma.

\section{Immunocytochemistry}

Antibodies. The antibody to glutamate used in the present study was the generous gift of Drs. C. Toomim and P. Petrusz. The antibody was raised by injection of rabbits with the amino acid coupled by glutaraldehyde to keyhole limpet hemocyanin (Hepler et al., 1986). The antibody has been further characterized by Toomim et al. (1986) and Hepler et al. (1988).

In a preliminary study (Johansen et al., 1987), we reported the labeling of neuromuscular endings with an aspartate-specific antiserum (Toomim et al., 1986; Hepler et al., 1988). However, additional control expcriments performed in our system suggested that this aspartate antiserum does not stain specifically in Drosophila tissue, and therefore, these data are not included in this study. In contrast, the glutamate antiserum used was highly specific for glutamate (Fig. 1, $A, B$ ).

Immunoblots. Glutamate, aspartate, and GABA were coupled to BSA with glutaraldehyde and purified by spin column centrifugation with Sephadex G25. The BSA-conjugate was applied in droplets $(1 \mu \mathrm{l}$ of a $0.5 \mathrm{~mm}$ BSA-conjugate solution) to nitrocellulose paper, which was then processed for peroxidase immunocytochemistry. The primary antiserum dilution used was $1: 1000$. In some experiments the primary antiserum was preincubated $2-6 \mathrm{hr}$ at $4^{\circ} \mathrm{C}$ with $0.5 \mathrm{~mm}$ of BSA amino acid conjugate.

Bodywall immunocytochemistry. Third instar larvae were dissected in Ca-free insect saline by a dorsal incision through the bodywall and pinned out flat in Sylgard-coated dishes. All internal organs wcrc rcmoved, exposing the inner surface of the bodywall muscles. The preparations were fixed in $4 \%$ paraformaldehyde, $0.2 \%$ glutaraldehyde in PBS, pH 7.4, for $1 / 2-1 \mathrm{hr}$, blocked in PBS with $10 \%$ NGS for $1 \mathrm{hr}$, and incubated overnight in primary antibody (1:400 dilution) made up in PBS with $0.2 \%$ Triton X-100 (TBS) and $1 \%$ NGS at room temperature. After washing in TBS for $1 \mathrm{hr}$ the bodywalls were incubated in HRPconjugated secondary antibody for $2 \mathrm{hr}$ before reaction with 3,3'-diaminobenzidine $(0.05 \%)$ and $\mathrm{H}_{2} \mathrm{O}_{2}(0.003 \%)$. The final preparations were rinsed in PBS and cleared and mounted on slides in 5\% n-propyl gallate glycerol (Giloh and Sedat, 1982). In some experiments rhodamine-conjugated secondary antibody was used and the preparations mounted in $n$-propyl gallate glycerol.

Immunoelectron microscopy. For electron microscopic immunocytochemistry, 4 different bodywall preparations were dissected and reacted with primary antibody and peroxidase-conjugated secondary antibody as described above. The tissue was then postfixed in $2 \% \mathrm{Os}_{2} \mathrm{O}_{4}$ in PBS for $1 \mathrm{hr}$, dehydrated through an alcohol series, and embedded in Spurr's. Gold sections were cut with glass knives and stained lightly with lead citrate before viewing with a Zeiss $10 \mathrm{EM}$.

CNS immunocytochemistry. Antibody reactions were performed on sectioned ganglia since whole-mount immunocytochemistry was unsuccessful on nervous systems fixed with the minimal level of glutaraldehyde that was required to preserve antigenicity. Ganglia dissected from third instar larvae were fixed in $0.1 \%$ glutaraldehyde $/ 4 \%$ parafor- maldehyde in $100 \mathrm{~mm}$ PBS, pH 7.4 for $1 \mathrm{hr}$. Following dehydration in an alcohol series, the ganglia were briefly stained with $0.1 \%$ eosin yellow in ethanol, cleared in methyl benzoate, and transferred through toluene for Paraplast embedding. The $8 \mu \mathrm{m}$ sections, affixed to glass slides with polyvinyl alcohol/vinyltriethoxysilane (Fink, 1987), were incubated at room temperature for $1 \mathrm{hr}$ in 10\% NGS in PBS and for $2 \mathrm{hr}$ in a $1: 1000$ dilution of the primary antiserum in 1\% NGS in PBS. Visualization of primary antibody staining was done using the avidin-biotin goat anti-rabbit IgG-HRP method (Vector $\mathrm{ABC}$ kit), with diaminobenzidine at $0.5 \mathrm{mg} / \mathrm{ml}$ and $\mathrm{H}_{2} \mathrm{O}_{2}$ at $0.01 \%$. The stained sections were dehydrated in an alcohol series, cleared with xylene, and mounted in DPX mountant (Gurrs, Poole, UK).

\section{Electrophysiology}

Current-and voltage-clamp. Bodywall preparations were dissected with the CNS attached so that fine $\mathrm{AgCl}$ hook electrodes could be placed under the peripheral nerves for stimulation. To limit spontaneous contractions of the muscles, the abdominal part of the ganglion was crushed with forceps. Intracellular recording from individual muscles was done with $2.5 \mathrm{M} \mathrm{KCl}$-filled glass capillary electrodes with resistances of from 5-20 Ms. In some experiments, the muscle cells were voltage-clamped with a Dagan 8500 two-elcctrode voltage-clamp operated at a fixed bandwidth of $1 \mathrm{kHz}$. Membrane current was measured by a virtual ground circuit connected to the bath via an $\mathrm{AgCl}$ pellet electrode. Current and voltage traces were photographed directly from the oscilloscope display. Good spatial voltage control was readily obtained in the larval muscle fibers since they are relatively short cylinders that are virtually isopotential (Ian and Jan, 1976a; Wu and Haugland, 1985). For desensitization experiments, the saline solutions in the experimental chamber were exchanged through a continuous perfusion system. All experiments were done at room temperature $\left(21-23^{\circ} \mathrm{C}\right)$.

Iontophoresis. Drosophila larvae were dissected in $0 \mathrm{~mm} \mathrm{Ca}, 6 \mathrm{~mm}$ $\mathrm{Mg}$ saline. The bodywalls were sectioned along the dorsal midline and secured on a glass "coffin" slide with fine steel pins. The bodywalls werc viewed with a $40 \times$ water-immersion objective using differential interference contrast optics, and the image was displayed with a final magnification of $2000 \times$ on a video monitor. The screen image of the muscle fibers and the electrodes was printed by a digital copy unit, giving a map location of sensitive regions on the muscle fiber. Using the video optics up to 3 iontophoresis electrodes could be manipulated to within $1 \mu \mathrm{m}$ of each other, eliminating the need for multiple-barrel electrodes. The separate electrodes for each test substance also reduced the chance of artifactual release of transmitter. The intracellular recording was made with $2.5 \mathrm{M} \mathrm{KCl}$-filled microelectrodes with resistances of 10-20 M . Electrodes were filled with solutions of $\mathrm{Na}$ glutamate or $\mathrm{Na}$ aspartate at concentrations ranging from 0.1 to $1.0 \mathrm{M}$. The $\mathrm{pH}$ of the solutions was adjusted to approximately $\mathrm{pH} 8$ with $\mathrm{NaOH}$. Iontophoresis electrodes typically had resistances between 100 and $200 \mathrm{M} \Omega$. For intracellular injection of glutamate and aspartate by iontophoresis, each muscle fiber was filled by the application of 200 negative pulses of $500 \mathrm{msec}$ duration and of $100 \mathrm{nA}$ amplitude.

\section{Results}

\section{Antibody specificity}

The glutamate antiserum used in this study is highly specific for glutamate. Toomim et al. (1986) and Hepler et al. (1988) showed by immunoblot analysis that the serum had no or only minimal $(\leq 10 \%$ ) cross-reactivity with related amino acids (including GABA, glutamine, aspartate, and asparagine), which were selected for testing on the basis of their chemical similarity and possible biological relevance. However, with the possibility of both glutamatergic and aspartergic inncrvation of the bodywall muscles (Irving and Miller, 1980b), it was of special importance in this study to make certain that the glutamate antiserum did not cross-react with aspartate in the tissue. Therefore, we further tested the specificity of the antiserum on immunoblots before and after preabsorption of the antibody with BSA-conjugates of glutamate and aspartate (Fig. 1). Figure $1 A$ shows that the glutamate antibody reactivity was almost completely eliminated by preabsorption of the antisera with glu-BSA, whereas incu- 


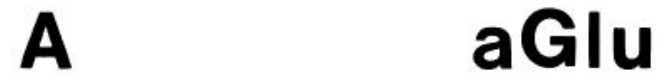

preabsorption

\section{Glu-BSA Asp-BSA}
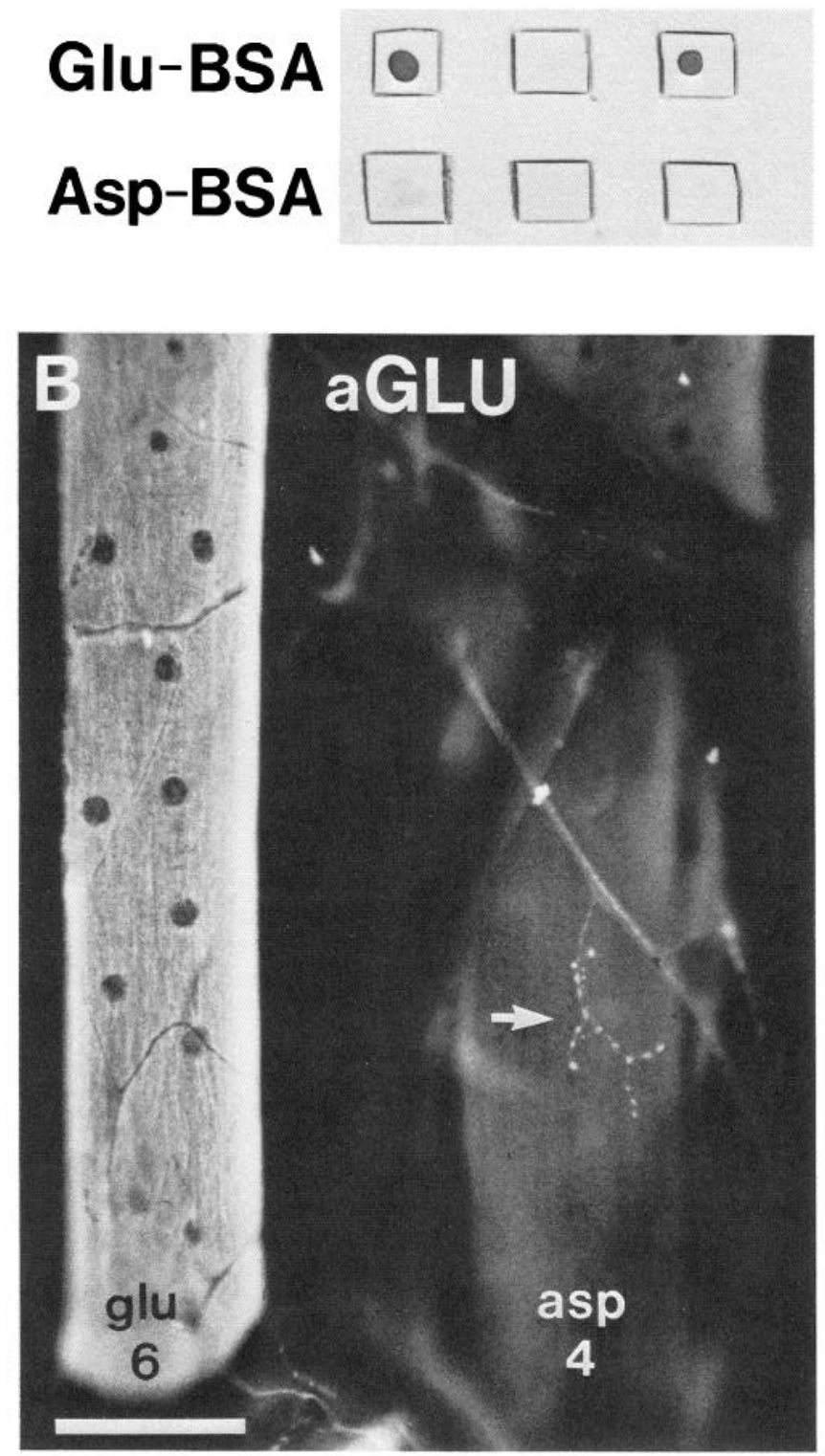

Figure 1. Specificity of the glutamate antiserum. $A$, Glutamate and aspartate were conjugated to BSA (Glu-BSA and $A s p-B S A)$ with glutaraldehyde and applied to nitrocellulose paper. The conjugates on the immunoblots were incubated with glutamate antiserum $(a G l u)$ and bound antibody demonstrated with peroxidase labeled secondary antibody. In addition, blots are shown where the antiserum was preabsorbed with Glu-BSA and Asp-BSA conjugate, respectively. $B$, Muscles 6 and 4 in Drosophila were iontophoretically injected with approximately equal amounts of glutamate $(g l u)$ and aspartate (asp) respectively, incubated in glutamate antiserum $(a G L U)$, and processed for immunocytochemistry. The glutamate-injected muscle 6 shows clear labeling, whereas the aspartate-injected muscle 4 is stained only slightly above background. Note the strongly stained neuromuscular ending (arrow) on the aspartate-injected muscle 4 (right hemisegment). Rhodamine secondary antibody. Scale bar, $50 \mu \mathrm{m}$. bation with asp-BSA leaves the immunoreactivity of the antiserum for glutamate intact. Moreover, the weak cross-reactivity between anti-glutamate and aspartate was completely eliminated by preincubation of the glutamate antiserum with aspBSA (Fig. 1A). Consequently, preabsorption of the glutamate antiserum with asp-BSA before histological experiments was done routinely. As a further test of antibody specificity, we iontophoretically injected different bodywall muscle fibers with equal amounts of glutamate and aspartate, respectively, and processed the preparations for immunocytochemistry. Figure $1 B$ shows a muscle 6 that was injected with glutamate and is clearly stained by the glutamate antiserum, whereas the muscle 4 injected with aspartate is labeled only slightly above background. This result, together with the immunoblot analysis, makes it unlikely that the glutamate antiserum after preincubation with asp-BSA had any significant cross-reactivity with aspartate in the fixed tissue. In addition we confirmed the result of Hepler et al. (1988) that the glutamate antiserum had no cross-reactivity with GABA (not shown), an inhibitory transmitter candidate at insect neuromuscular junctions.

\section{Glutamate immunoreactive neuromuscular synapses and neurons}

\section{Morphology of glutamatergic muscle innervation}

The musculature of Drosophila larvae is remarkably simple, consisting of up to 31 discrete unicellular muscle fibers per hemisegment. Each muscle fiber is uniquely identifiable, and many are segmentally repeated. Five segment-specific patterns exist for segments $T_{1}, T_{2}, T_{3}, A_{1}-A_{7}$, and $A_{8}$, respectively (Campos-Ortega and Hartenstein, 1985). In the following account of glutamatergic muscle innervation, the identification and numbering of individual muscle fibers in Drosophila are from Crossley (1978) and Anderson et al. (1988) and in Calliphora from Crossley (1965). Most of the illustrated muscle fibers are from the left hemisegment, with anterior being at the top of the figures; however, a few muscle fibers (Figs. $1 B, 3 A$, and muscle 4, 6, and 7 in Fig. 4) are from the right hemisegment.

With the glutamate antiserum we observed immunoreactive nerve endings on all bodywall muscle fibers in every thoracic and abdominal segment, as well as on the intersegmental muscles of the larval mouthparts. The staining obtained was specific and limited to neuronal endings; no immunoreactivity with the muscle fibers or trachea was observed. Figure 2 shows the staining of muscle fibers in a whole-mount bodywall preparation reacted with the glutamate-specific antiserum. Each of the dorsolateral muscle fibers $(1,2,3,9$, and 10$)$ is innervated by immunoreactive endings given off by the anterior branch of the segmental nerve, which runs ventral to dorsal near the middle of each segment. In some cases, as exemplified by muscle fiber 2 , the muscle fibers were innervated by 2 separate branches from the nerve (Fig. 2, arrows). While this could be due to either 2 collateral branches of the same axon or to dual innervation from 2 glutamatergic neurons, we never found any examples of muscle fibers innervated by more than 2 separate nerve branches. After examining a large number of preparations $(>150)$, we conclude that all muscle fibers in every segment are innervated by at least one glutamate immmunoreactive axon.

Figures 3 and 4 illustrate the morphology of immunoreactive nerve terminals and their distribution on identified muscle fibers. The multiterminal innervation was initiated at characteristic nerve entry points on the internal muscle surface (Table 1) from where several varicose nerve fibers branched off (Figs. 2, 


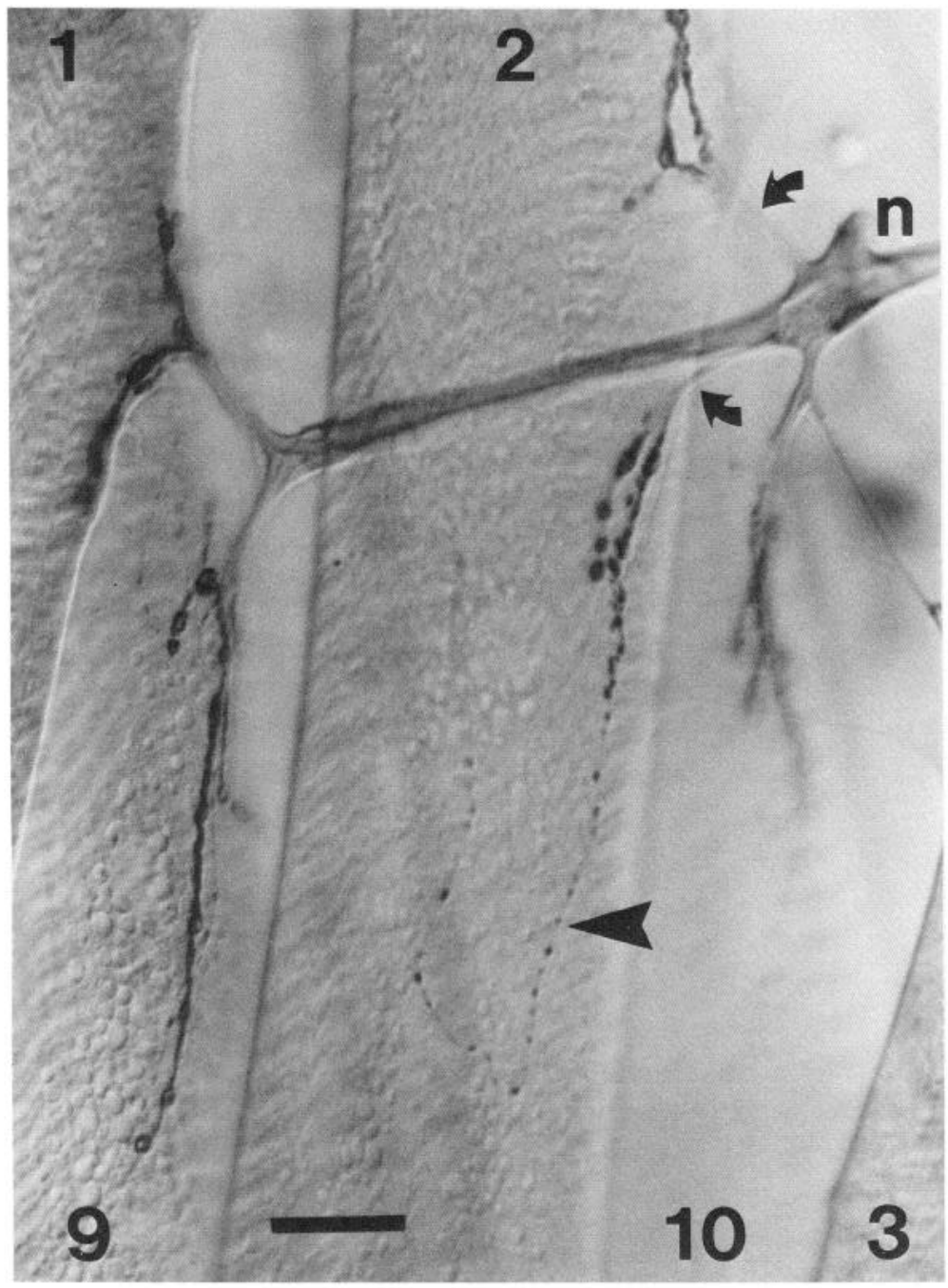

Figure 2. Glutamate immunoreactive motor endings on identified muscle fibers $(1,2,9$, and 10) in Drosophila. The anterior segmental nerve $(n)$ gives off branches with varicose endings to all the muscle fibers. The curved arrows indicate 2 different branches of the nerve innervating the same muscle fiber. The arrowhead points to the thin Type II varicose endings of less than $2 \mu \mathrm{m}$. Peroxidase immunocytochemistry. Scale bar, $25 \mu \mathrm{m}$
3). While most nerves contact the muscle fiber near its midpoint, for some muscle fibers, as in the case of muscle 5 , the innervation was initiated near the apodeme insertions.

Two types of varicose nerve projections could be distinguished on the basis of their morphology and the size distribution of their varicosities (boutons). One type of nerve process (Type I) had branches usually located close to the point of innervation. These endings had a broad size distribution of relatively large boutons ranging up to $8 \mu \mathrm{m}$ (mean diameter, $3.1 \pm 1.6$ $\mu \mathrm{m} ; \pm \mathrm{SD}, n=521)$. In contrast, the other type of nerve process (Type II) consisted of very thin neurites $(<0.5 \mu \mathrm{m})$, with a narrow distribution of smaller boutons with a mean diameter of only $1.4 \pm 0.6 \mu \mathrm{m}( \pm \mathrm{SD}, n=214)$. Type II processes unlike Type I endings often projected over the entire internal muscle surface (see Fig. 4). Although Type I and Type II endings were often distinct from the site of nerve entry, it remains to be determined whether they represent different branches of the same axon or whether they are derived from separate motoneurons. Thus, the distinction made between Type I and Type II processes does not imply that they necessarily belong to different axons, but rather represents an operational classification for descriptive purposes.

A comparison of the innervation of muscle fibers both be- tween segments and from different preparations revealed a remarkably specific and stereotyped branch morphology of especially the larger Type I processes associated with particular muscle fibers. That is, the different identified muscle cells had well-defined, distinguishable branching and innervation patterns of glutamate immunoreactive nerve endings. In this paper we report in detail on the stereotyped innervation of muscle 1 , $4,5,6,7,12$ and 13, which were selected as representative examples. The innervation pattern of these muscle fibers is illustrated by the microphotographs in Figures 3 and 5, by the camera lucida drawings in Figure 4 (which show the distribution of nerve endings over the entire surface of the muscle fibers), and by the summary of innervation features in Table 1.

The differences in the innervation pattern of these muscle cells are very striking. For example, muscle fibers 12 and 13 are each innervated by a single discrete nerve branch from the posterior segmental nerve with the varicose nerve endings fanning out in a characteristic pattern (Figs. $3 A, 4$ ). On muscle 12 between 2-3 Type I processes branch off both anteriorly and posteriorly for up to $100 \mu \mathrm{m}$ from an innervation point on the side of the muscle fiber. One long Type II process projects posteriorly over the entire length of the muscle fiber. On muscle 13, however, the innervation is initiated on the central part of the muscle 


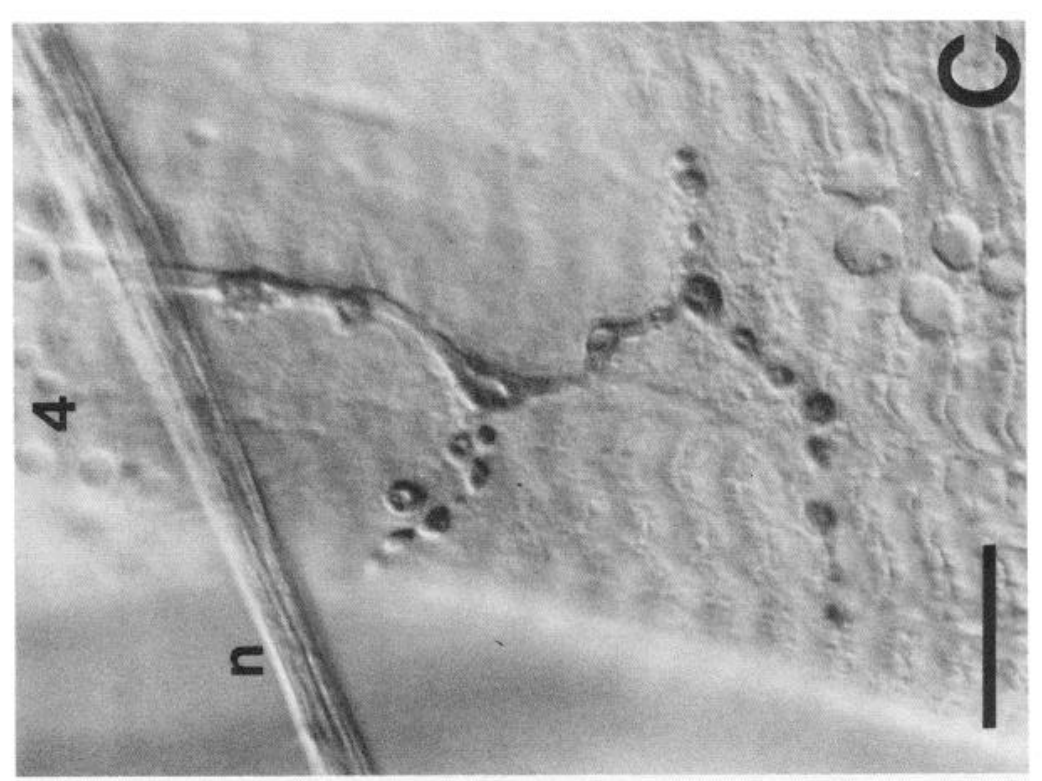

分窎

동

产岕

율 로

호요

กั ฐ

范范

ป⿻

运

党

ธี 을

ถ⿻

틍층
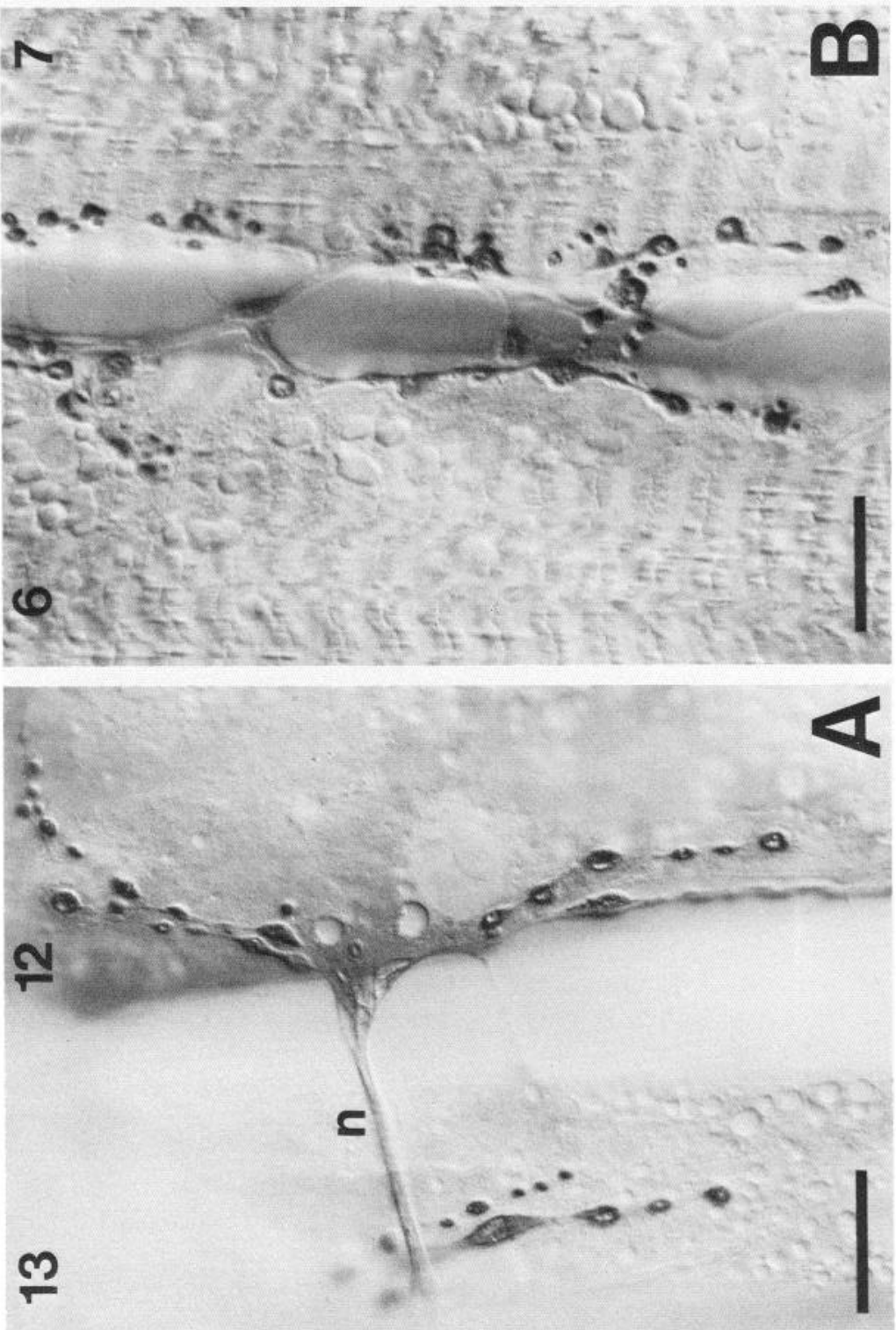

흐

त.

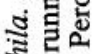

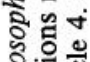

今.

픔염

홍ㅎํ

.

뭉요

.

ज्

oñ

记.

प0工

员嵒总

氙总

s

든.

o.

氠

品 을

苍证

$\infty \infty$

हैं

농

密

E

E

s.

吨

吾

的过

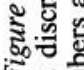



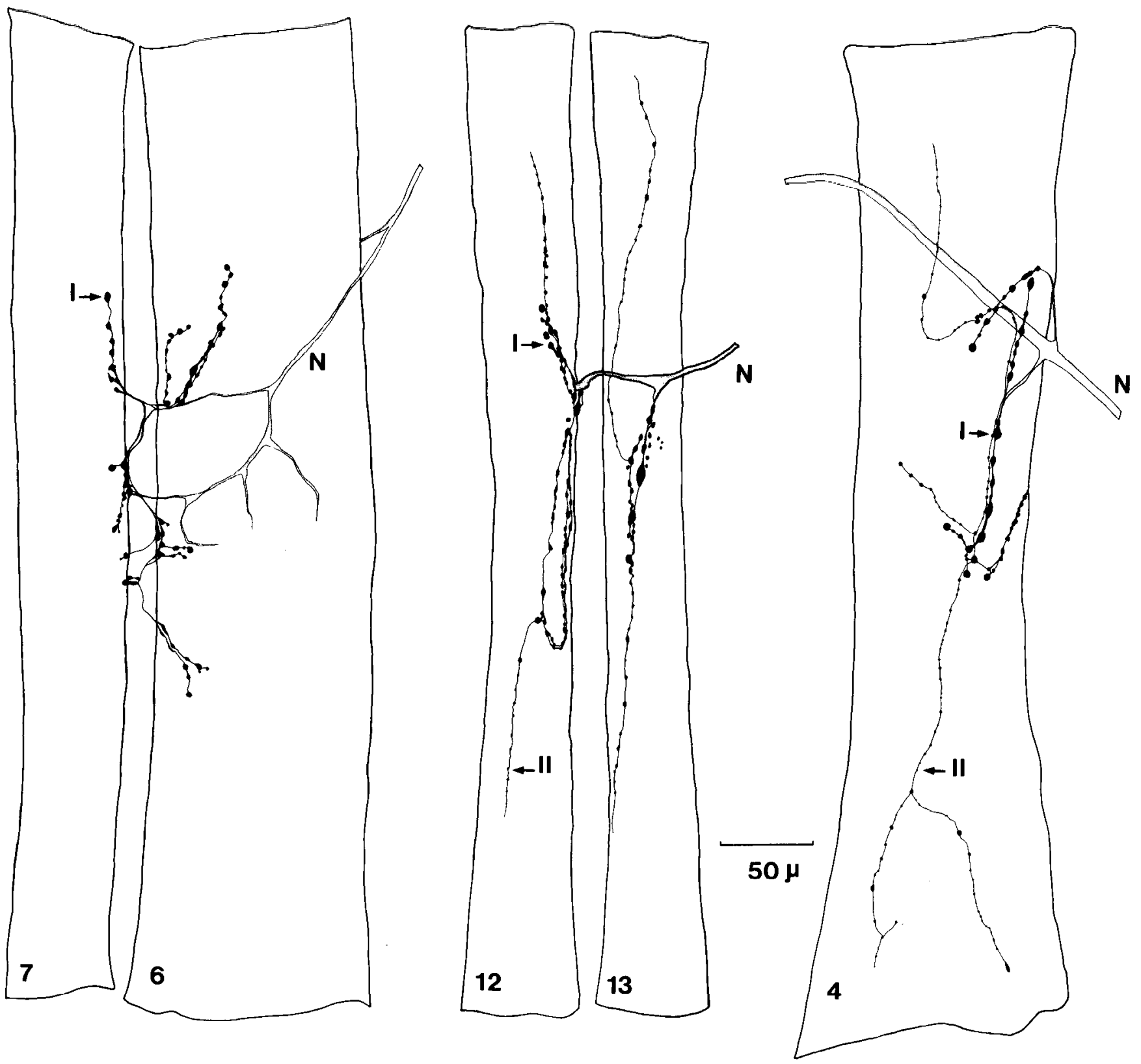

Figure 4. Camera lucida tracings of glutamate-immunoreactive endings on identified muscle fibers in Drosophila. Examples of the entire innervation pattern of muscles 4, 6, 7, 12, and 13 are shown. Examples of Type I and Type II endings are indicated by arrows. Note the extensive ramifications of the thin Type II endings on muscle 4 and their absence on muscles 6 and 7 . Muscles 4, 6, and 7 are from the right hemisegment. The nerve $(N)$ is shaded.

fiber and the Type I processes are directed only posteriorly. In contrast to these patterns of innervation, muscles 6 and 7 are innervated by one nerve branch from the posterior segmental nerve going to both muscles. This nerve branch gives off axons projecting longitudinally along the cleft between the muscles. Close examination shows that both muscle fibers are innervated by common axons which can be seen to give off alternating terminals to the 2 muscles. Muscles 6 and 7 are not innervated by the thin Type II nerve endings, only by Type I endings (Figs. $3 B, 4)$ and thus have a broad size distribution of relatively large boutons with a mean diameter of $3.0 \pm 1.5 \mu \mathrm{m}( \pm \mathrm{SD}, n=218$; Fig. $6 A$ ). Muscle 4 , on the other hand, which is innervated by the anterior branch of the segmental nerve, typically has only a relatively short stretch of innervation by Type I nerve endings and has a greater proportion of the thin Type II endings (Fig. 4). This is clearly reflected in the total size distribution of boutons on muscle 4 , where $73 \%$ of the boutons are less than $2 \mu \mathrm{m}$ in contrast to muscle 6 and 7 , where only $26 \%$ are less than 2 $\mu \mathrm{m}$ (Fig. $6 A$ ). The difference in size distribution of the boutons between Type I and Type II nerve endings on muscle 4 is very pronounced, as illustrated in Figure $6 \mathrm{~B}$. Moreover, the size distribution between boutons from Type I endings on muscle 4 is indistinguishable from the distribution on muscles 6 and 7 (Fig. $6 \mathrm{C}$ ). This suggests that different muscle fibers exhibit uniformity in their Type I process morphology. Muscle 4 is also characterized by being innervated from 2 separate subbranches from the anterior segmental nerve (Fig. 4, Table 1). Figure 5 shows the high degree of stereotypy of the short segment of Type I 


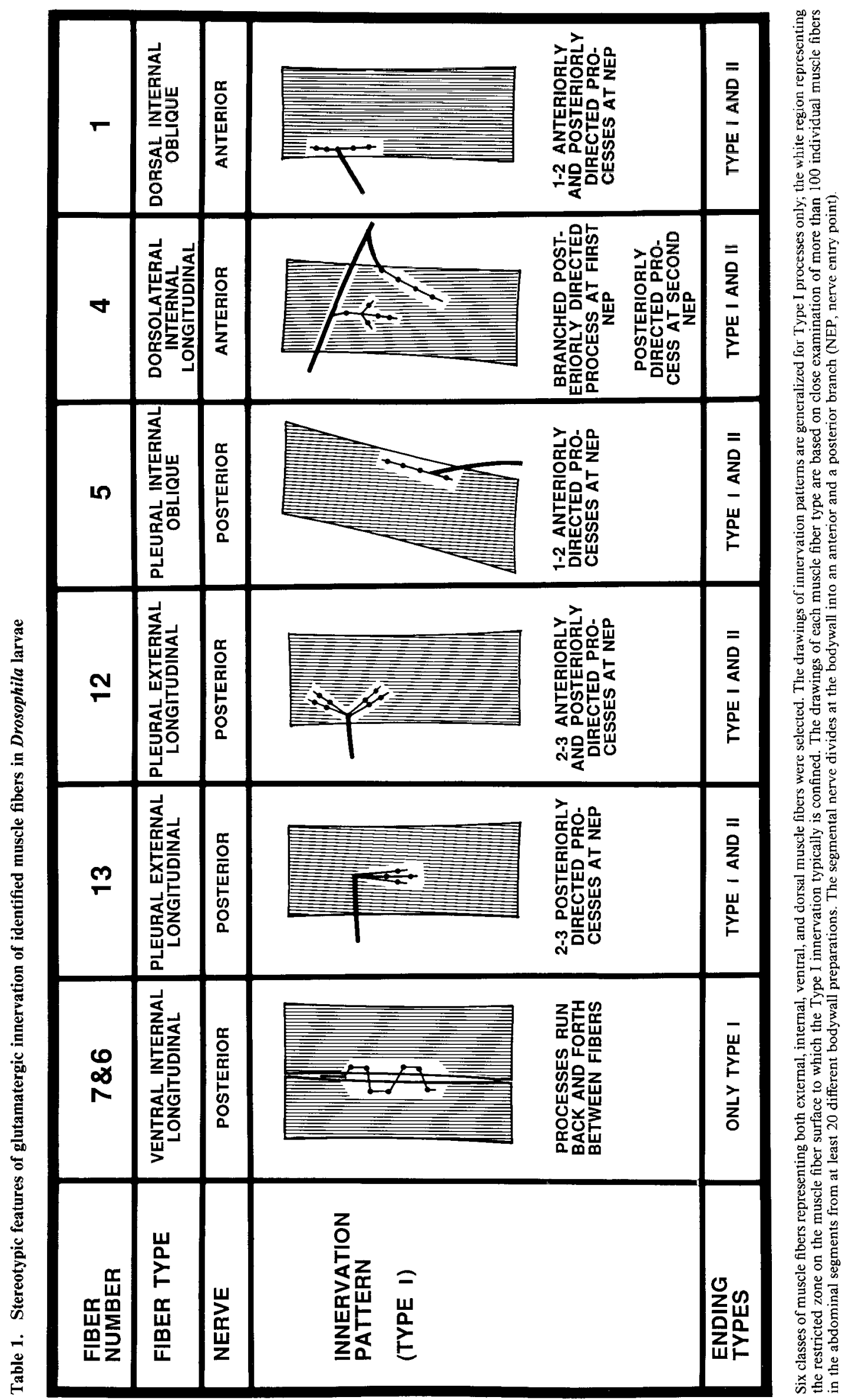



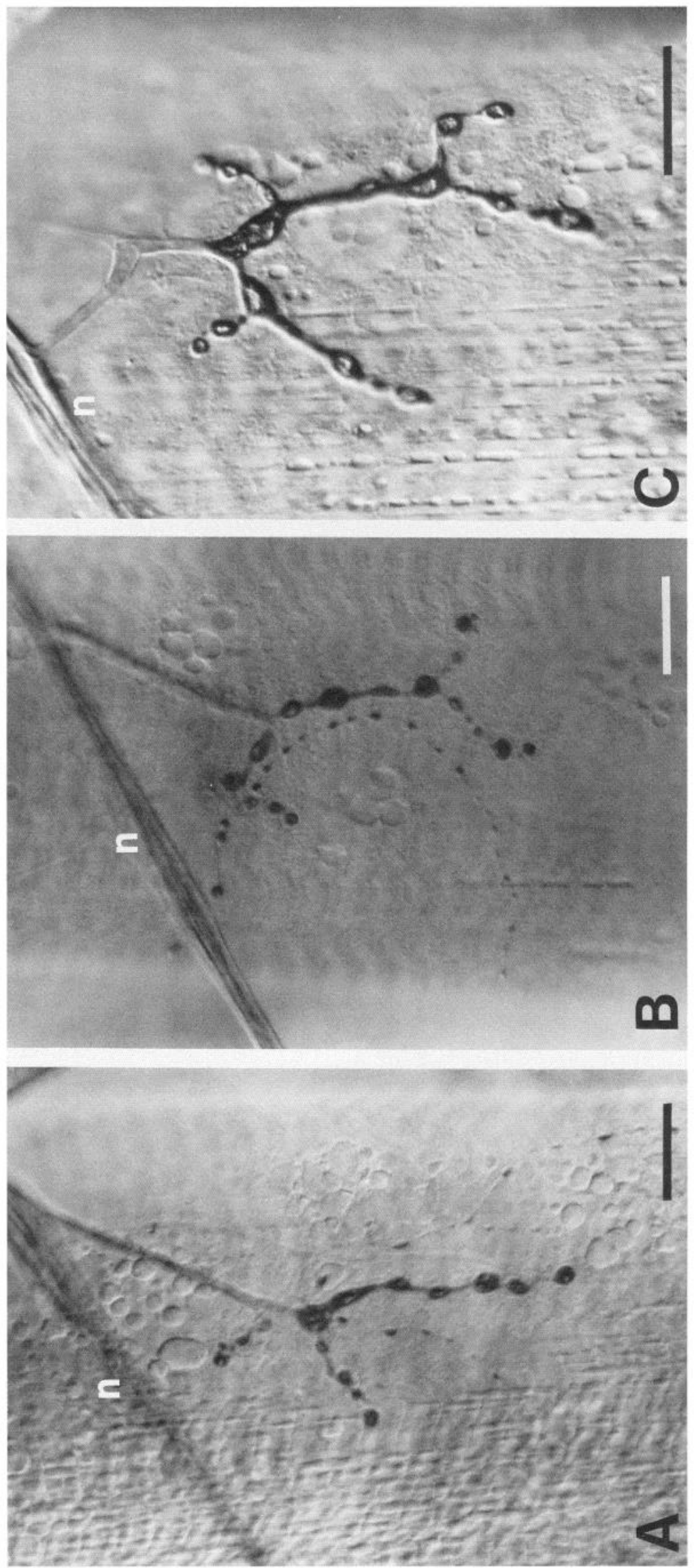

옳

园

声

콜

흠

옥.은

t

ติะ

密

녕

춘

递

인

की 进

哂

今.

논 

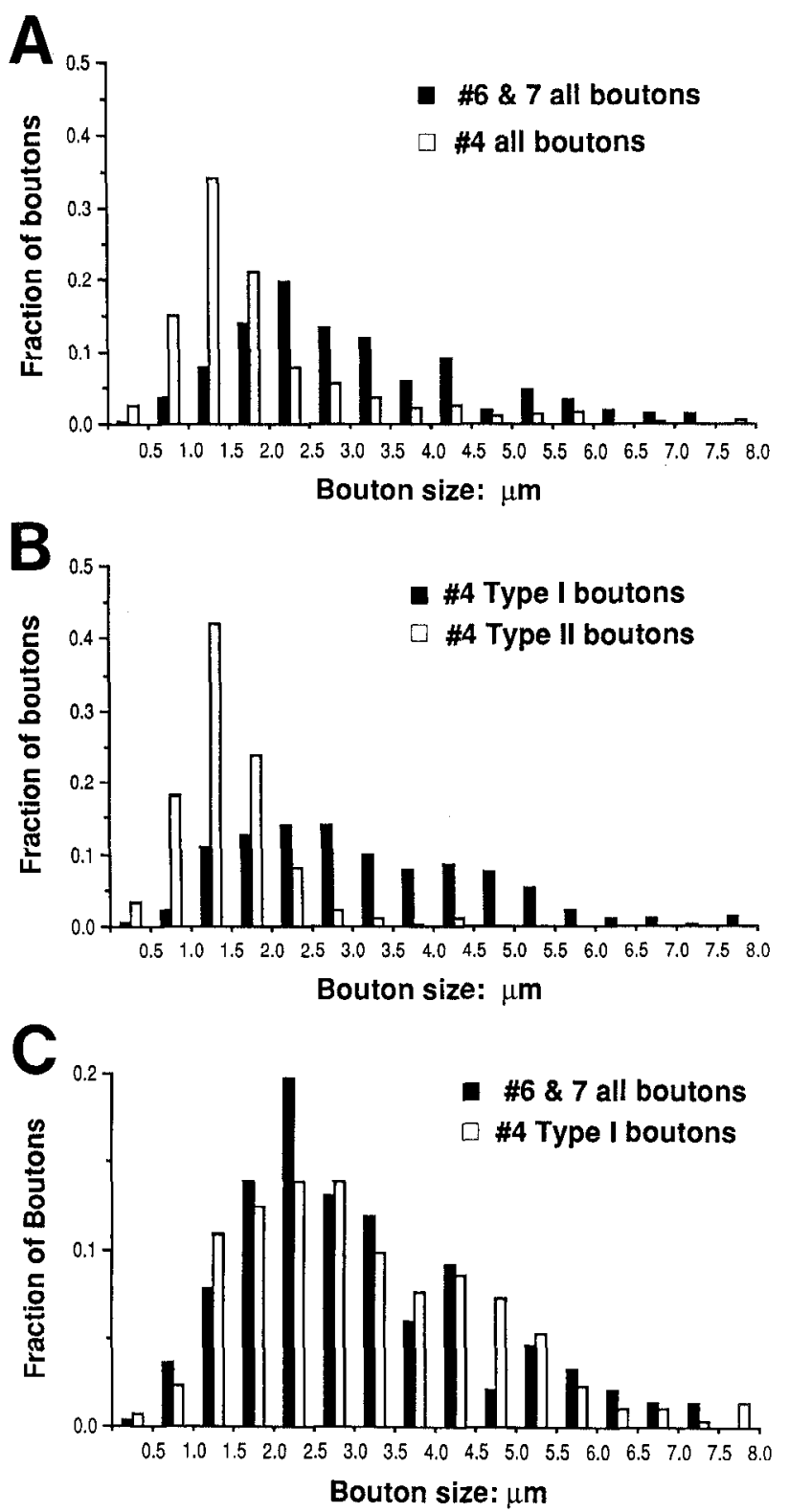

Figure 6. Size distributions of boutons from Type I and Type II nerve processes are distinct. $A$, Comparison between the size distribution of the entire population of boutons from 4 pairs of muscles 6 and 7 and of 4 muscle 4's that were randomly selected. The largest axis of the boutons were measured in each instance. Muscle 4 is characterized by having a large fraction of small boutons in contrast to muscles 6 and 7 , which are characterized by having a broad distribution of larger boutons. The mean diameter for boutons on muscle 6 and 7 was $3.0 \pm$ $1.5 \mu \mathrm{m}( \pm \mathrm{SD}, n=218)$ and $1.8 \pm 1.3 \mu \mathrm{m}( \pm \mathrm{SD}, n=279)$ on muscle 4. $B$, Comparison between the size distributions of boutons of Type I and Type II nerve endings on muscle 4 . In this experiment Type I and Type II nerve endings were visually identificd and their bouton size distribution determined separately. The Type II nerve endings show the expected narrow distribution of small boutons, whereas the Type I endings had a clearly different, much broader distribution of larger boutons. The mean diameter for boutons from Type I nerve endings was $3.2 \pm 1.7 \mu \mathrm{m}( \pm \mathrm{SD}, n=303)$ and $1.4 \pm 0.6 \mu \mathrm{m}( \pm \mathrm{SD}, n=214)$ for the Type II nerve endings. $C$, Comparison between bouton sizes of nerve endings on muscle 4 and on muscles 6 and 7 showing the 2 distributions to be very similar. In all cases the area under the histogram has been normalized to unity. innervation on muscle 4 from 3 different muscle fibers (compare also with Figs. $1 B$ and $3 C$ ). The stereotypic features of the innervation of these muscle fibers are further defined and summarized in Table 1. It should be noted that although the precise branching patterns on individual muscle fibers showed a fair amount of variation, the listed features were always conserved, making the innervation pattern of these muscle cells uniquely recognizable.

\section{Ultrastructure of immunoreactive varicosities}

To demonstrate that the observed glutamate immunoreactivity was associated with neuromuscular synapses, we examined the antibody staining of the nerve endings at the ultrastructural level. Glutamate antibody-stained, peroxidase-reacted bodywall preparations were prepared for electron microscopy and ultrathin sections made through areas with stained varicosities. Figures $7, A, B$, show 2 such preparations, where one is heavily and the other more lightly stained. The immunoreactivity is confined to membrane-bound endings and is associated with $30-50 \mathrm{~nm}$ vesicles (Fig. $7 \mathrm{~B}$ ) typical of neuromuscular junctions in Drosophila larvae (Jan and Jan, 1976a). Mitochondria in the endings are unstained. The nerve endings are enveloped by a subsynaptic reticulum of invaginated sarcolemma, which is also a characteristic feature of dipteran neuromuscular junctions (Osborne, 1967; Hardie, 1976; Jan and Jan, 1976a). The synaptic cleft between the axolemma and the sarcolemma is evident in Figure $7 A$. Thus, the described immunoreactive endings exhibit all the known features of synaptic terminals of the neuromuscular junction in Drosophila larvae. These results indicate that the 30-50 nm synaptic vesicles are glutamate immunoreactive and provide new independent evidence in support of an endogenous transmitter role for glutamate.

\section{Iontophoretic mapping of glutamatergic responses}

Jan and Jan (1976b) showed by iontophoresis that depolarizing potentials in Drosophila larval muscle fibers could be elicited by glutamate and that nerve terminals could be located by focal application of the glutamate transmitter. We used this technique to map glutamate-sensitive receptor sites on several different muscles using high-resolution video microscopy in order to compare their localization with the pattern of innervation revealed by immunoreactivity. Fifty-eight muscle fibers from 18 Drosophila larvae were assayed for their sensitivity to iontophoretically applied glutamate. Seven different internal and external muscle fiber types were tested in segments $T_{2}-A_{5}$. An iontophoretic potential due to glutamate could be generated in all the muscle fibers examined. The size of the potentials varied proportionally with the distance of the electrode from the muscle fiber surface (Fig. 8C) and with the amount of iontophoretic current applied (Fig. 8D).

The mapping of the muscle fibers revealed small responsive sites, with the iontophoretic potentials dropping off sharply at approx. 5-10 $\mu \mathrm{m}$ on each side of a sensitive site (Fig. $8 B$ ). The hot-spots on specific muscle fibers mapped to restricted and stereotyped regions, which suggests that these muscles do not possess randomly distributed extra junctional receptors to glutamate (Jan and Jan, 1976b). As an example, Figure $8 A$ shows a video image of the surface of muscle 6 upon which sensitive sites have been mapped by applying constant test pulses of glutamate in $10 \mu \mathrm{m}$ increments (Fig. $8 B$ ). The glutamate-responsive sites were concentrated along the lateroventral region of the muscle fiber, with the remaining muscle surface being 


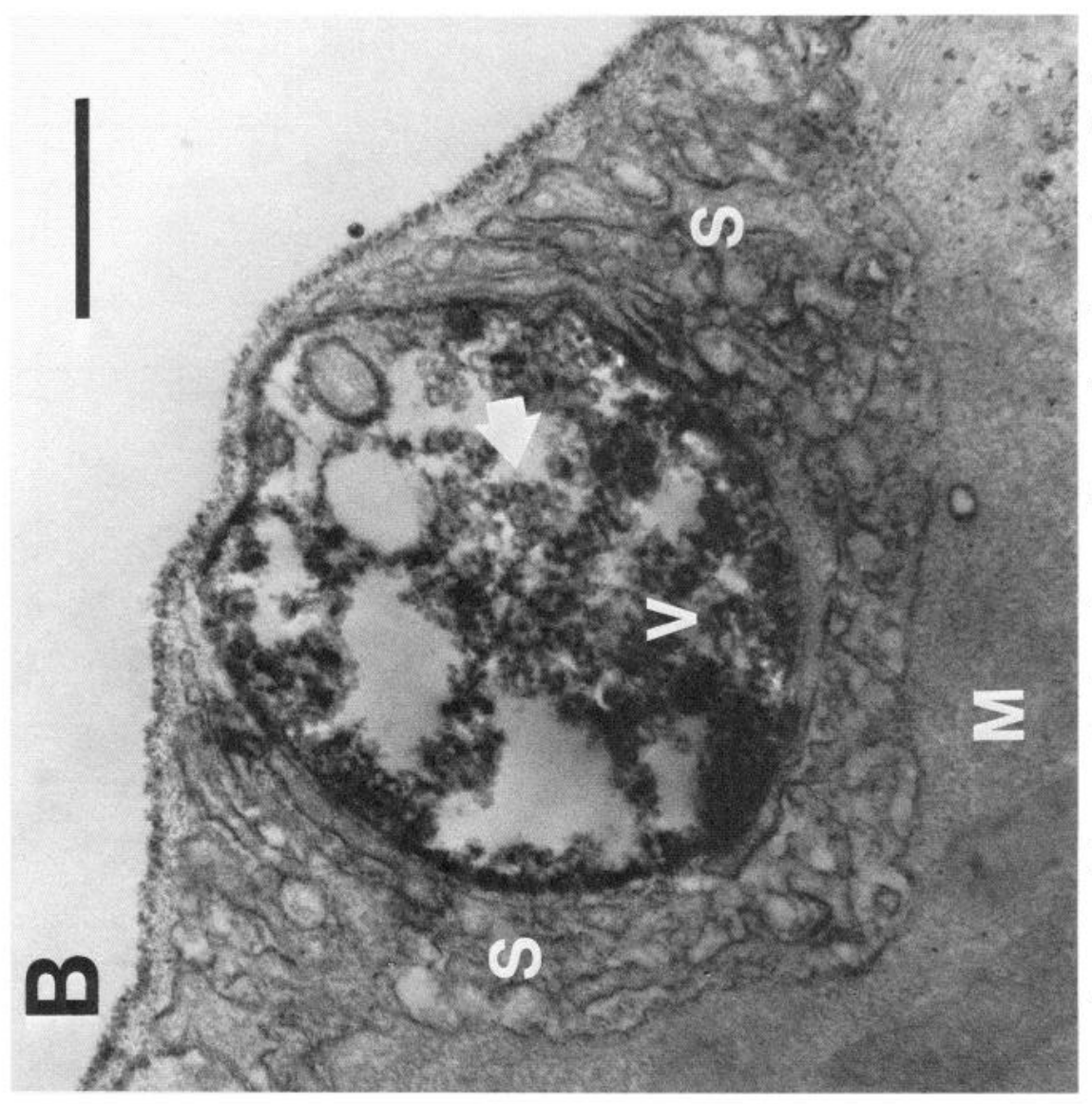

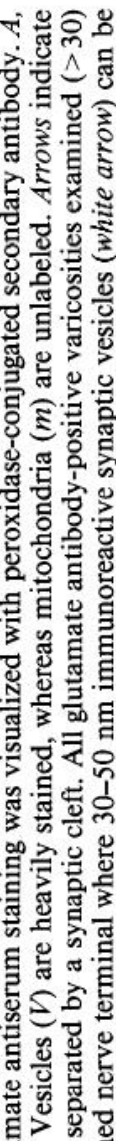

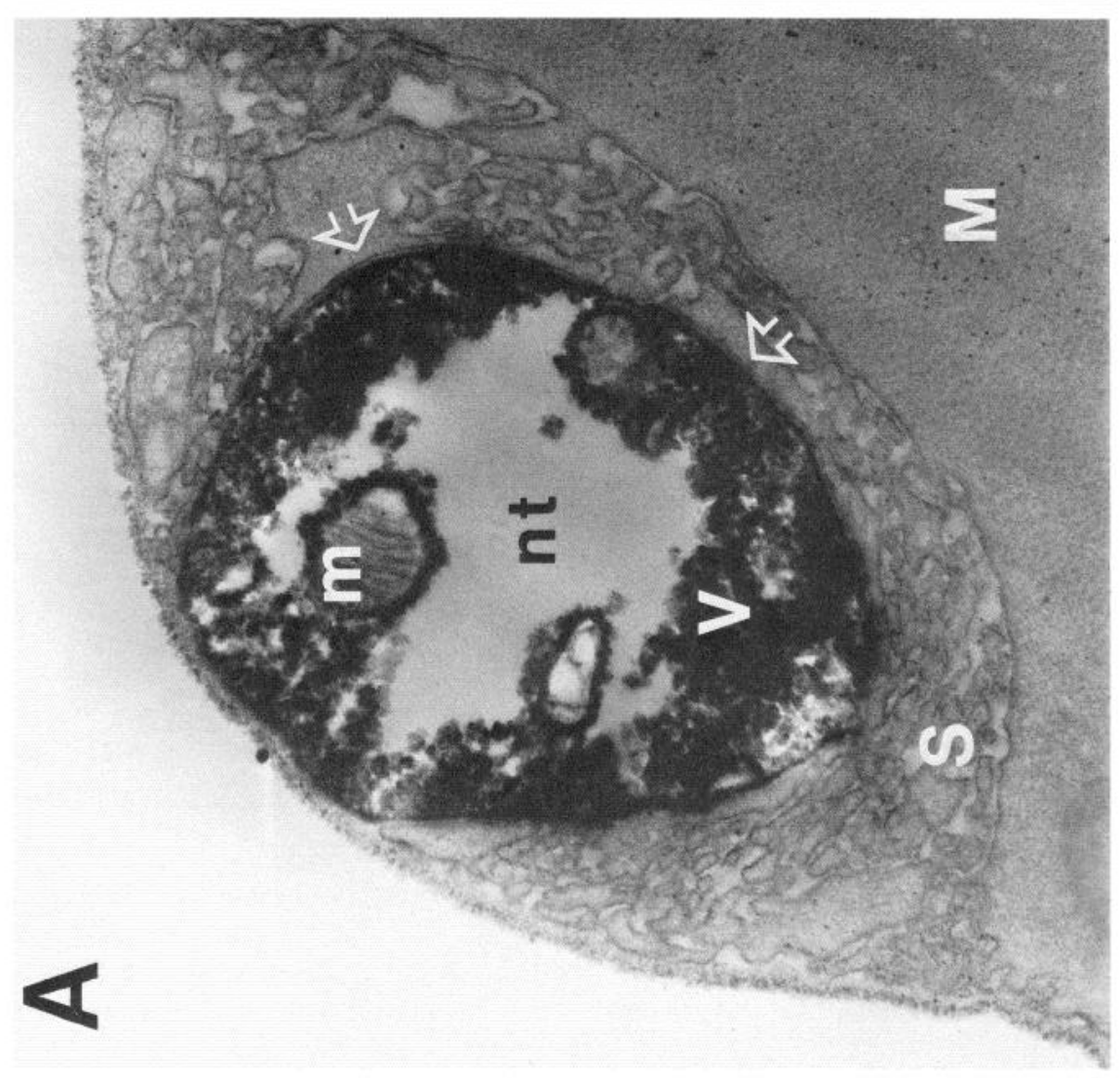
它的.

目要总

.

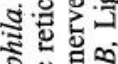

S.0.

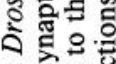

을.

象南

흐을

o

을 웅

인.․․

完㣢

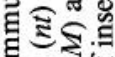

증

ज证

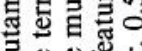

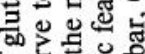

웡.

记战

或응

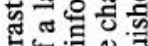

5 o

焉

슬

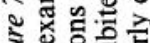

政考. 

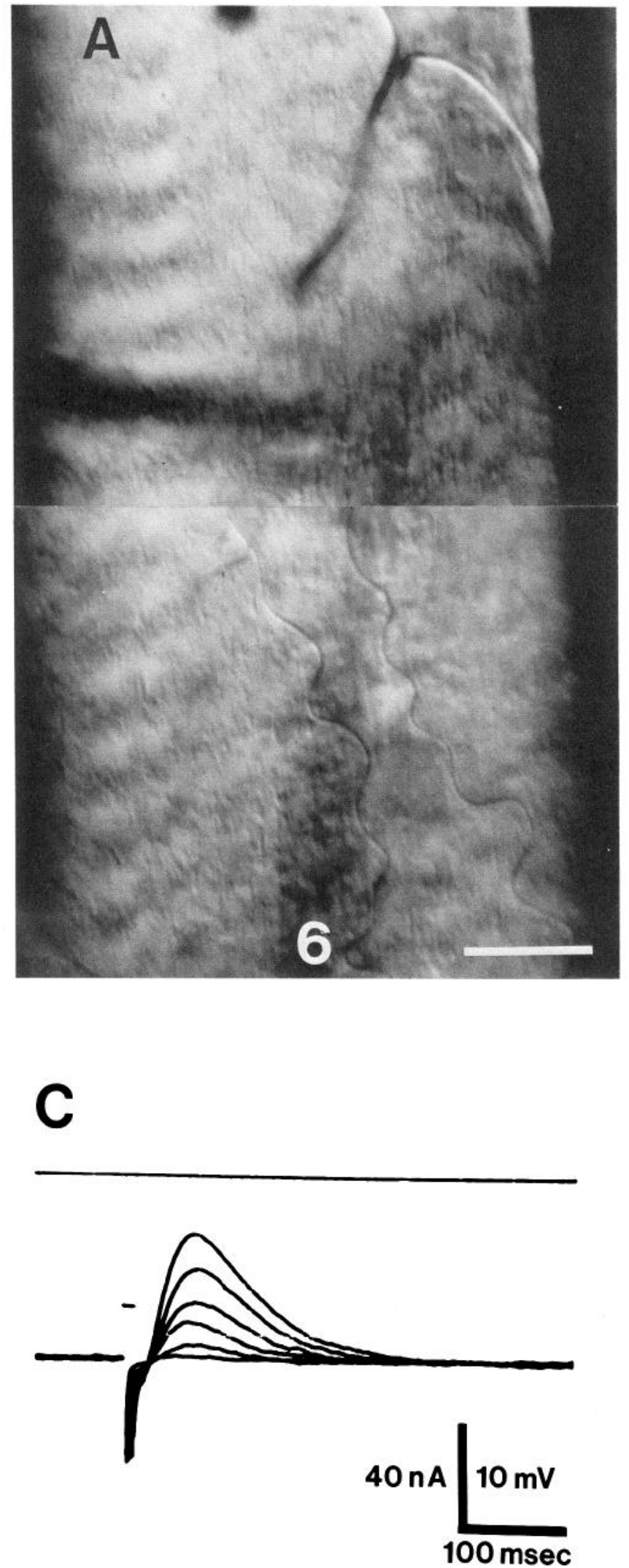

B
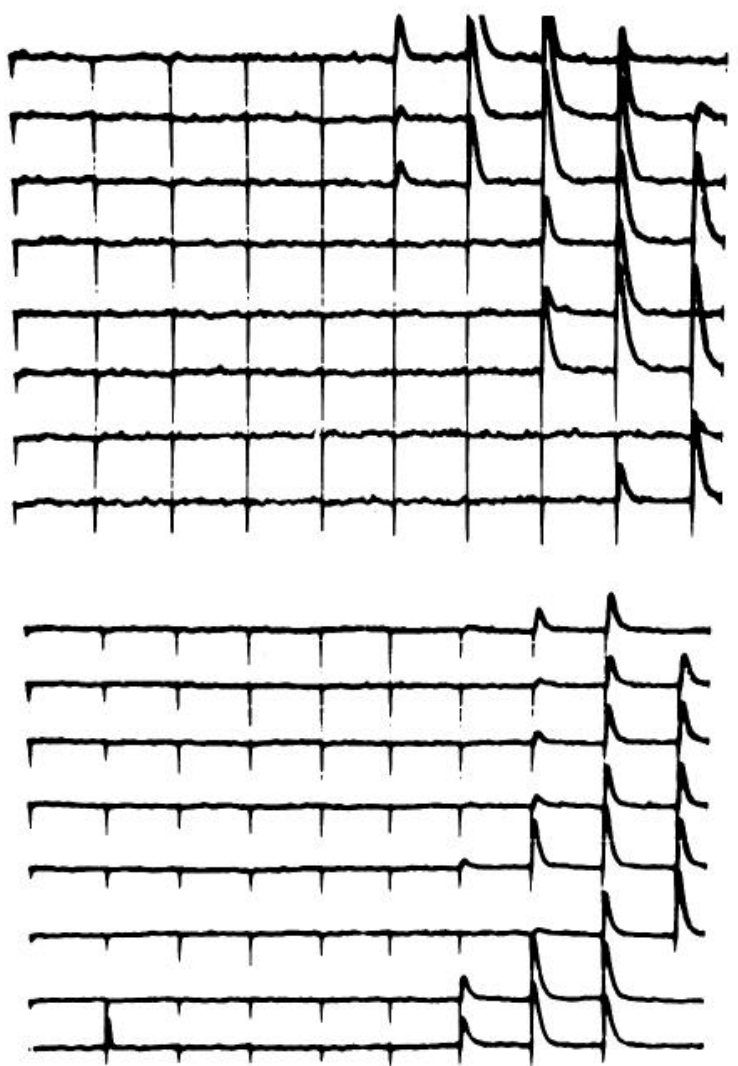

D

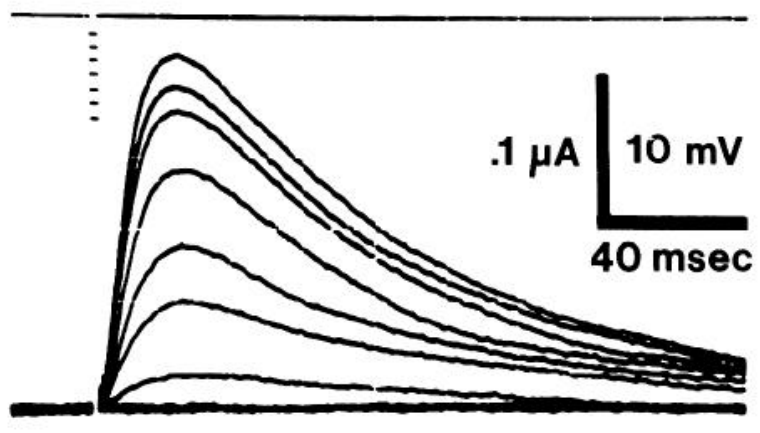

Figure 8. Iontophoretic mapping of glutamatergic synapses in Drosophila. A, Video image using Nomarski optics of the surface of muscle fiber 6 upon which glutamate-sensitive sites have been mapped as shown in $B$. Motor endings on the living muscle cannot be reliably resolved by light microscopy even using enhanced-contrast video techniques. Scale bar, $20 \mu \mathrm{m}$. B. Sensitive sites on the muscle shown in $A$ were mapped in response to a constant iontophoretic pulse of glutamate while the electrode was moved across the muscle surface. The muscle surface was scanned in approximately $10 \mu \mathrm{m}$ increments as indicated by the spacing of the stimulus artifacts. Glutamate-responsive sites were found to be confined to the 
unresponsive. These results are in good correspondence with the pattern of immunocytochemical staining, which showed that muscles 6 and 7 are innervated only in the narrow cleft region where the 2 muscle fibers lie juxtaposed (Figs. $3 B, 4$ ). In addition, we performed detailed mapping studies of muscle fibers 4,12 , and 13 , which were found to have clearly defined hot spots in the muscle surface regions where the Type I immunoreactive endings were localized. This confirms that the pattern of glutamate-responsive sites for these muscle fibers corresponds to the pattern for glutamatergic endings obtained by immunocytochemistry. Consequently, these results suggest that receptors sensitive to glutamate are colocalized with glutamate-containing nerve endings, providing additional evidence for a transmitter role for glutamate.

\section{Glutamate immunoreactive neurons in the CNS}

Glutamate immunoreactivity was also examined in the CNS of third instar larvae in order to demonstrate the presence of glutamatergic neurons potentially responsible for the peripheral staining on the muscle fibers. Incubation of paraffin sections with the glutamate antibody revealed strong neuropil and cell body staining in the brain hemispheres and ventral ganglia. As shown in Figure 9, the entire neuropil was consistently stained; however, only a subset of the total CNS cell population was immunoreactive. These cell bodies, ranging from 3 to $6 \mu \mathrm{m}$ in size, were found in clusters throughout the CNS, with some indication that they were organized as repeated units in a bilaterally symmetric fashion. Two prominent features of the cell body staining were the presence of larger, strongly stained neurons located both dorsally and ventrally at the posterior end of the ganglion and segmentally iterated pairs of cells that lay beneath the neuropil along the midline (Fig. 9). The fact that only a subset of neurons was stained argues against the antibody immunoreactivity being a consequence of metabolic glutamate present in the general neuronal population. Note also the absence of glutamate antibody-stained cells (Fig. 9C) in the ventral zone of undifferentiated neurons derived from the larval neuroblasts (Truman and Bate, 1988). On the basis of these results it seems likely that some of the selectively glutamate-immunoreactive neurons may be motoneurons innervating the peripheral muscles.

\section{Aspartate as an excitatory transmitter}

The present and previous experiments (Jan and Jan, 1976a, b) provide good evidence that glutamate is present in nerve terminals of Drosophila larval neuromuscular junctions and that receptors sensitive to glutamate are colocalized with the glutamate-containing nerve endings. However, the question remains whether glutamate is a transmitter at both the slow and the fast junction or if one of the junctions uses another excitatory neurotransmitter. It is currently well established that at least 2 axons innervate larval muscle fibers based on (1) morphological evidence from methylene blue and reduced silver nitrate staining (Hardie, 1976); (2) that there are 2 axons with different thresholds to nerve stimulation (Jan and Jan, 1976a; Hardie and Os- borne, 1977; Irving and Miller, 1980a, b); and (3) that the reversal potential at the 2 junctions differs (Jan and Jan, 1976b; Irving and Miller, 1980b). We have addressed this question by conducting electrophysiological experiments in both Drosophila and Calliphora larvae.

\section{Iontophoresis and desensitization of aspartate and glutamate}

In order to determine whether an excitatory physiological transmitter function for aspartate could be demonstrated, we performed iontophorcsis cxpcriments as previously described for glutamate. However, in contrast to the robust and readily obtained glutamate potentials, we never observed iontophoretic potentials due to aspartate on any of the muscle fibers examined ( $n=41$ in 13 larvae) despite careful mapping and the use of currents up to 50 times that for a threshold glutamate response. As a further test we performed desensitization experiments for nerve-evoked slow and fast potentials under current- and voltage-clamp in muscle fibers from both Drosophila and Calliphora larvae. The segmental muscle pattern of Calliphora larvae is homologous to that of Drosophila. Identical results from several types of muscle fibers were obtained in both species; however, more experiments were done with Calliphora larvae due to their larger and more robust potentials. Muscle fibers in which stimulation of the innervating nerve elicited 2 potentials with different thresholds and time course were voltage-clamped in control Ringer's. The preparation was then perfused with either glutamate- or aspartate-containing saline. Figure 10 shows that $5 \mathrm{~mm}$ glutamate completely eliminated both the slow and the fast EPSC in the muscle fiber. The desensitization was reversible (not shown). In contrast, both the fast and the slow EPSC persisted after incubation for more than $1 / 2 \mathrm{hr}$ in $5 \mathrm{~mm}$ aspartate (Fig. 11). The contractions of the muscle fibers in response to nerve stimulation also remained in $5 \mathrm{~mm}$ aspartate. Similar results were obtained for aspartate concentrations up to $10 \mathrm{mM}$. These results from 2 other species of dipteran larvae are consistent with those of Robinson (1981) obtained in Lucilia larvae and suggest that glutamate is a transmitter at both neuromuscular junctions and make a transmitter role for aspartate unlikely.

\section{Discussion}

Glutamate as a transmitter at the neuromuscular junction Jan and Jan (1976a, b) proposed, on the basis of electrophysiological evidence, that glutamate was a likely candidate for a neuromuscular transmitter at the bodywall muscles of Drosophila larvae. However, in their experiments they could not determine whether glutamate itself was the endogenous transmitter or only a closely related agonist to the receptor. In this paper we have extended thesc obscrvations by an immunocytochemical approach and demonstrated with a glutamate antiserum that all bodywall muscles receive at least one glutamateimmunoreactive neuromuscular ending. This conclusion is based on several lines of evidence indicating that the observed immunoreactivity was a direct consequence of endogenous glutamate localized in neuromuscular synaptic terminals. (1) Im-

\footnotetext{
ventrolateral edge of the muscle fiber in good accordance with the pattern of motor endings obtained by glutamate immunocytochemistry (Figs. $3 B, 4$ ). $C$. Iontophoretic potentials (bottom trace) in response to constant glutamate pulses (top trace) with the electrode being withdrawn in $2 \mu \mathrm{m}$ increments from 2 to $12 \mu \mathrm{m}$ above the muscle fiber (\#7). D, Intracellular iontophoretic potentiais (bottom trace) in muscle fiber 6 in response to increasing current pulses (top trace) of glutamate (stimulus artifact has been removed).
} 


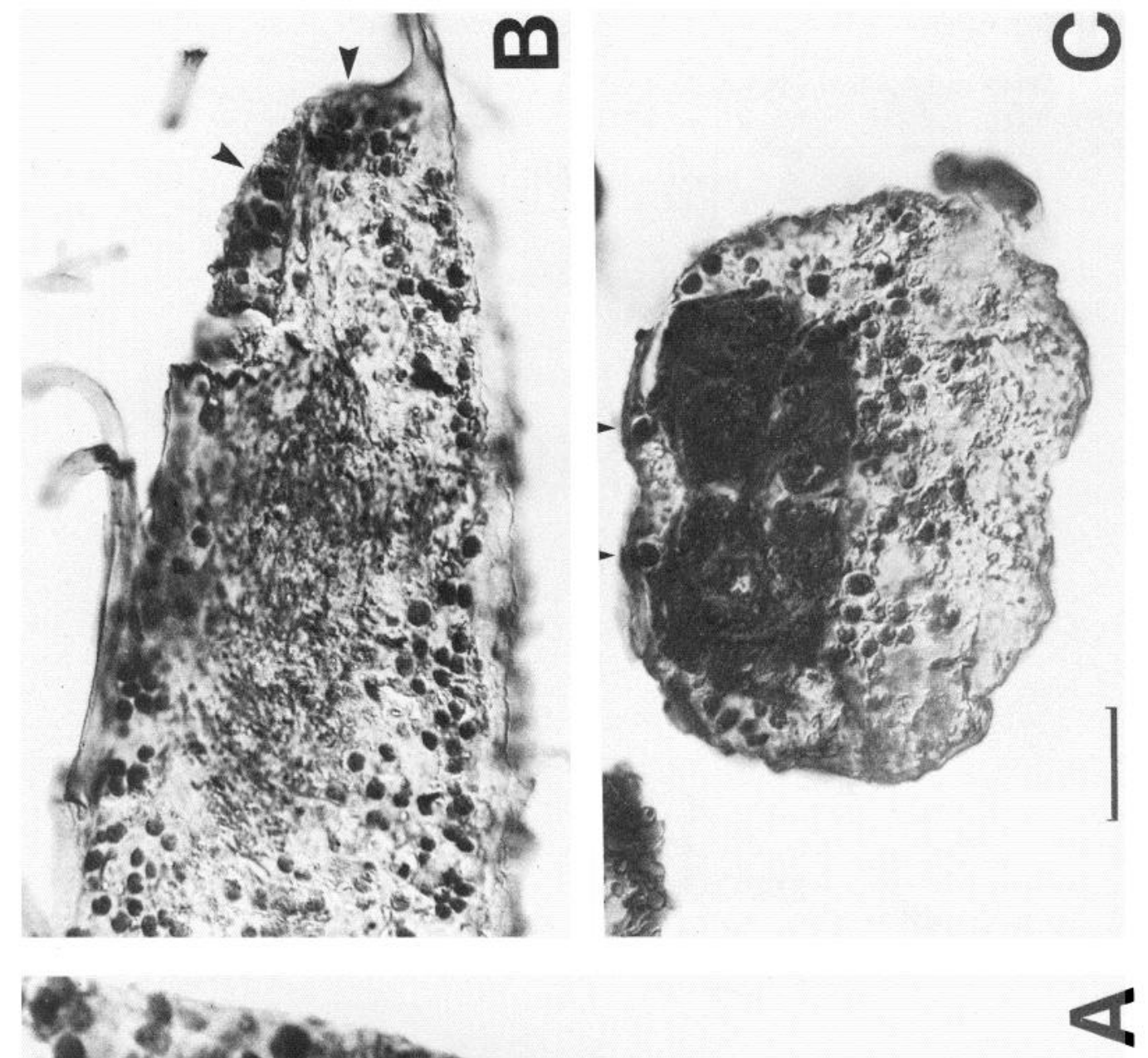

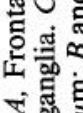

저으

를

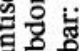

त $\pi$

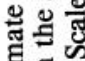

동

灵全官

突.

उen

政

急

음

政

을형

品

좁틀

80

윤

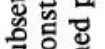

जै छ․․․

총. 똥

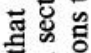

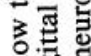

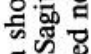

11 कू

品

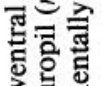

记

路

o

边 步

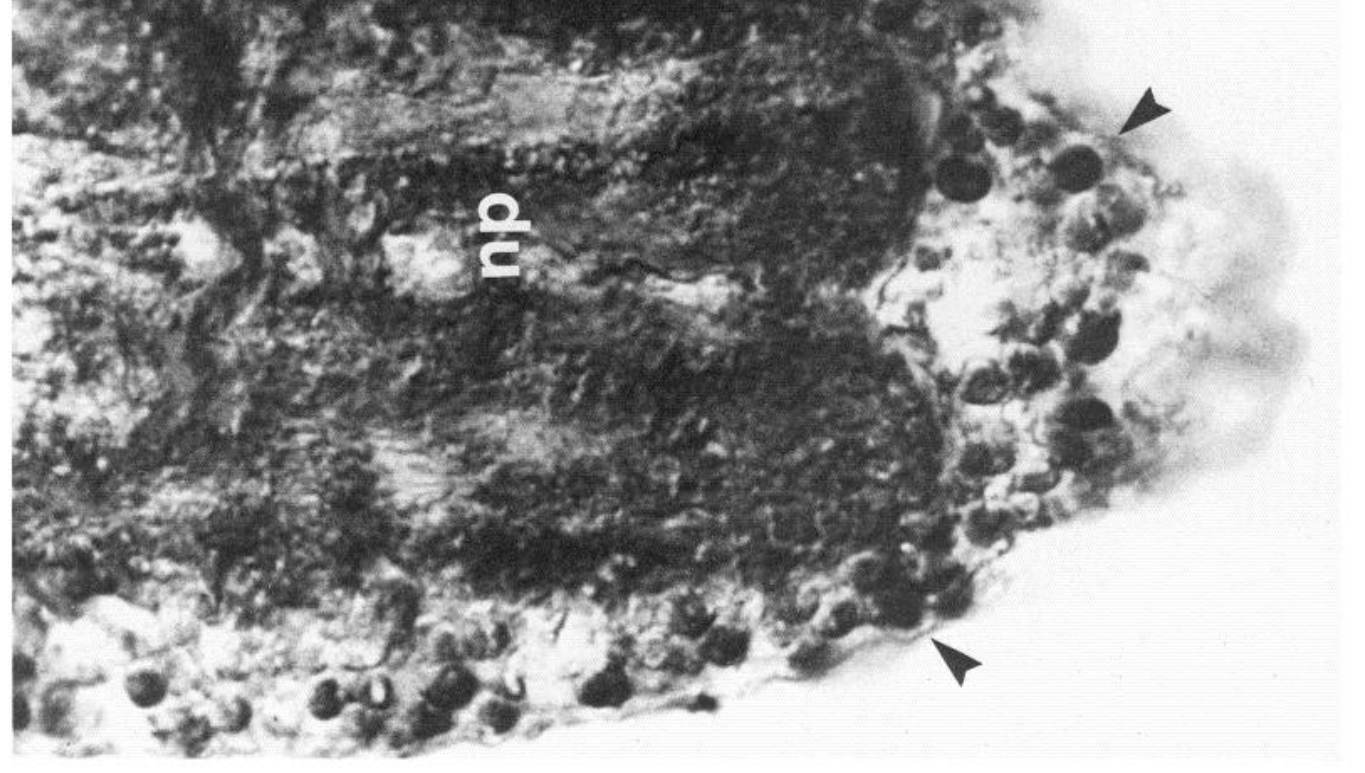

골

츤

.

D.

트를

है

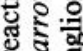

댕

츙

틍

\%

है

言哓

为路

乙管

․ के है

5

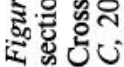




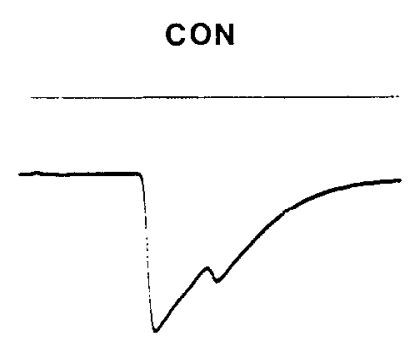

A

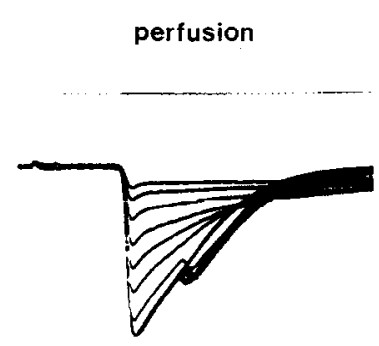

B

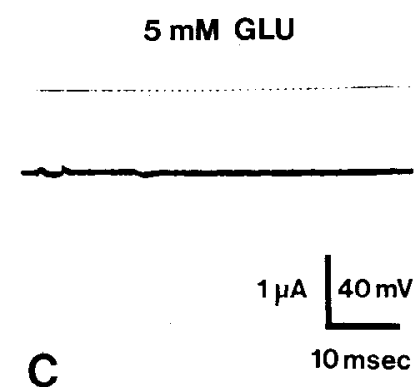

Figure 10. Desensitization of both the fast and the slow EPSC by glutamate in Calliphora. The records are from a 2 electrode voltage-clamp experiment on muscle $8 \mathrm{~b}$. The 2 superimposed EPSCs were evoked by suprathreshold stimulation of the segmental nerve. $A$, The slow and the fast EPSC in control insect saline $(C O N)$. $B$, Gradual reduction in size of the 2 EPSCs during perfusion with saline containing $5 \mathrm{~mm}$ glutamate. $C$, In $5 \mathrm{~mm}$ glutamate, both EPSCs were completely desensitized. The desensitization was reversible (not shown). Upper trace, voltage; lower trace, current.

munoblot assays of the antiserum showed it to be specific for glutamate with no or minimal cross-reactivity to related amino acids and dipeptides (this study; Toomim et al., 1986; Hepler et al., 1988). (2) Ultrastructural analysis showed that the glutamate immunoreactivity was associated with $30-50 \mathrm{~nm}$ vesicles located in synaptic structures surrounded by a subsynaptic reticulum that are characteristic features of insect neuromuscular endings. (3) Mapping with iontophoretically applied glutamate revealed that conductance-increasing receptors sensitive to glutamate were localized in a pattern coextensive with the immunoreactive endings. These results provide strong independent evidence that glutamate is present in presynaptic structures, meeting one of the major criteria for the demonstration of an endogenous transmitter. Furthermore, several enzymes (i.e., glutamate oxaloacetic transaminase, glutaminase, and glutamate dehydrogenase) thought to be important in the regulation of glutamate transmitter pools have been demonstrated to be present in Drosophila larvae (Chase and Kankel, 1987). However, to show conclusively that glutamate is a transmitter, release and uptake studies still have to be conducted in dipteran larvac. High-affinity uptakc of glutamate has becn demonstrated at other insect neuromuscular junctions (Faeder and Salpeter, 1970).

\section{Aspartate as a transmitter candidate at the slow junction}

Based on this evidence it is likely that glutamate is an excitatory transmitter at the neuromuscular junction in dipteran larvae. However, some ambiguity remains concerning the slow neuromuscular junction for which aspartate has been proposed as a transmitter candidate (Irving and Miller, 1980b). Our results from iontophoresis and desensitization experiments in Drosophila and Calliphora larvae and those of Robinson (1981) in Lucilia provide consistent evidence against a transmitter role for aspartate and suggest that glutamate is a neurotransmitter at both junctions. This conclusion is corroborated by the results in Drosophila of Jan and Jan (1976b). These workers also did not detect any physiological effects of aspartate, although the question of slow and fast junctions was not directly addressed in their experiments. Furthermore, recent results (Johansen et al., 1988) from double-labeling experiments with the glutamate antiserum and an antiserum to HRP, which labels the surface of the entire population of neurons and axons in Drosophila (Jan and Jan, 1982), suggest that all nerve endings innervating muscle fibers in Drosophila larvae are glutamatergic. The glutamate antiserum staining of neuromuscular endings in the pres- ent study is therefore likely to include innervation from both the fast and the slow axon. Although the contributions from these axons were not distinguishable by the immunocytochemical staining, an obvious possibility is that for some muscles the slow or the fast axon may be spccifically associated with cither Type I or Type II endings. However, one observation making this hypothesis less tenable is the fact that muscle 6 and 7 , which do receive dual innervation by electrophysiological criteria (Jan and Jan, 1976a), are innervated only by Type I nerve endings.

\section{Stereotyped morphology of glutamatergic innervation}

An important consequence of the glutamate-immunoreactive staining is that it permitted us to make the first detailed analysis of the morphology of innervating nerve endings and their projections over the entire cell surface of identified muscle fibers in Drosophila larvae. A remarkable property of the glutamateimmunoreactive endings revealed by this analysis is the high degree of structural stereotypy of nerve ending morphology associated with specific muscle fibers. The observed features of

\section{$5 \mathrm{mM}$ ASP}

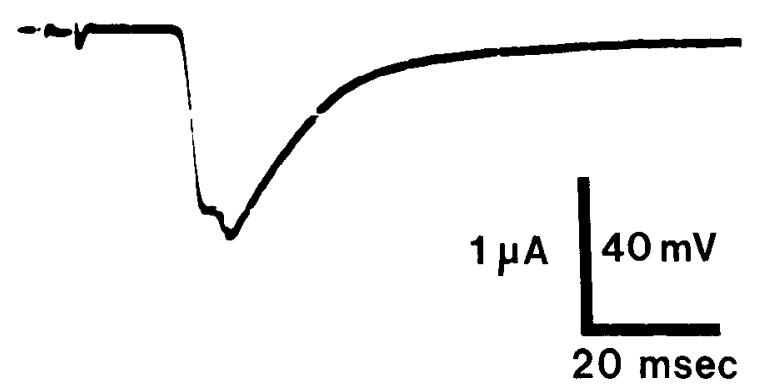

Figure 11. The slow and fast EPSCs persist in saline containing 5 mм aspartate. The record is from a voltage-clamp experiment on muscle $8 \mathrm{~b}$ in Calliphora. A biphasic EPSC could still be elicited by stimulation of the segmental nerve after half-hour incubation in $5 \mathrm{~mm}$ aspartate. Upper trace, voltage; lower trace, current. 
the glutamatergic innervation can be summarized as follows. (1) Characteristic regions of the cell surface of a specific muscle fiber are occupied by motor endings consisting of moderately branched multiterminal projections distributed on the internally facing surfaces of the fibers. (2) There are 2 types of motor endings. Type I, which have localized branching and relatively large varicosities ranging up to $8 \mu \mathrm{m}$, and the thinner Type II endings, which can extend over the cntirc intcrnal muscle surface and which have smaller varicosities of less than $2 \mu \mathrm{m}$. (3) Different identified muscle fibers have characteristic and distinguishable patterns of innervating branch morphologies. (4) The muscle fiber specific patterns of motor endings are reiterated by segmentally homologous fibers. (5) Most of the muscle fibers are innervated separately by either 1 or 2 branches coming off the segmental nerve. However, some muscle pairs, notably muscles 6 and 7, are innervated dually by the same axon which can be seen to give off alternative nerve terminals to both muscles. These muscles can form a functional unit as has been shown for the homologous muscles $6 \mathrm{~A}$ and $7 \mathrm{~A}$ in Calliphora by simultaneous intracellular recording of synchronous EPSPs in the 2 muscles (Hardie, 1976). A possible functional consequence of innervation by multiterminal stereotyped endings is that the postsynaptic potentials are evoked in a characteristic spatial fashion over each muscle fiber surface. This could be of physiological importance and be correlated to the kinetics of fiber excitation and to the contractile behavior of individual muscle cells.

The anatomy of the neuromuscular junctions labeled by glutamate immunocytochemistry described in the present study resembles in its general features the endings in dipteran larvae described by Hardie (1976) using methylene blue, by Anderson et al. (1988) using antisera to proctolin, and by Yoshikami and Okun (1984) using rhodamine 123 . The similarity of the varicose ending morphology described here to that of the vitally stained nerve endings of Yoshikami and Okun (1984) suggests that we have faithfully preserved the natural synaptic morphology using immunocytochemistry.

The pentapeptide proctolin is the only other neuromuscular transmitter convincingly documented in dipteran larvae. Anderson et al. (1988) demonstrated using HPLC and immunocytochemistry that Drosophila larval muscle fibers are innervated by axons containing the peptide. In contrast to glutamate, however, only a subset of the muscle fibers in segments posterior to segment $\mathrm{A} 2$ is innervated by proctolinergic synapses. It remains to be determined directly whether proctolin serves as a cotransmitter with glutamate, as is the case at the cockroach and grasshopper neuromuscular synapses (Adams and O'Shca, 1983; Worden et al., 1985).

Drosophila larvae are unusual in the high degree of stereotypy present in the organization of its musculature and neuromuscular junctions. A musculature composed of a small and apparently invariant number of muscle fibers suggests that each muscle fiber has a distinct identity and a specialized functional role. This implies that for a motor system made up of very few pre- and postsynaptic elements there must be a high degree of precision in the establishment of correct neuromuscular connections. The anatomical stereotypy of neuromuscular endings in Drosophila larvae discovered in this study is likely to reflect this aspect of connectivity. Another implication is that each muscle cell possesses regional cell-surface specializations or recognition molecules that are necessary for the different innervation patterns to be established. Important questions that can be addressed in this system on the single-cell level include the time course of establishment of the endings and their degree of plasticity during development, as well as the relative contributions made by the muscle fiber and the motoneuron in conferring the stereotyped features of the synapses.

In conclusion, we have provided both physiological and anatomical evidence that the bodywall muscle fibers of Drosophila larvae are innervated by motoneurons expressing glutamate as a transmitter. In addition, we provide evidence that the neuromuscular junctions are anatomically stereotyped with muscle fiber-specific branching patterns, which may reflect a high degree of precision in neuromuscular connectivity in this organism, as well as regional specialization of the muscle cell membrane. The ability to visualize the neuromuscular endings with the glutamate antiserum during embryogenesis in Drosophila may prove helpful for the further analysis of the mechanisms behind the establishment of such connections.

\section{References}

Adams, M. E., and M. O'Shea (1983) Peptide cotransmitter at a neuromuscular junction. Science 221: 286-289.

Anderson, M. S., M. E. Halpern, and H. S. Keshishian (1988) Identification of the neuropeptide transmitter proctolin in Drosophila larvae. Characterization of muscle fiber-specific neuromuscular endings. J. Neurosci. 8: 242-255.

Campos-Ortega, J. A., and V. Hartenstein (1985) The Embryonic Development of Drosophila melanogaster, Springer, Berlin.

Chase, B. A., and D. R. Kankel (1987) A genetic analysis of glutamatergic function in Drosophila. J. Neurobiol. 18: 15-42.

Crossley, A. C. (1965) Transformation in the abdominal muscles of the blue blowfly, Calliphora erythrocephala (Meig.), during metamorphosis. J. Embryol. Exp. Morphol. 14: 89-110.

Crossley, C. A. (1978) The morphology and development of the Drosophila muscular system. In The Genetics and Biology of Drosophila, Vol. 2b, M. Ashburner and T. R. F. Wright, eds., Academic, New York.

Faeder, I. R., and M. M. Salpeter (1970) Glutamate uptake by a stimulated insect nerve muscle preparation. J. Cell Biol. 46: 300-307.

Fink, S. (1987) Some new methods for affixing sections to glass slides. I. Aqueous adhesives. Stain Tech. 62: 27-33.

Giloh, H., and J. W. Sedat (1982) Fluorescence microscopy: Reduced photobleaching of rhodamine and fluorescein protein conjugates by n-propyl gallate. Science 217: 1252-1255.

Hardie, J. (1976) Motor innervation of the supercontracting longitudinal ventro-lateral muscles of the blowfly larva. J. Insect Physiol 22: 661-668.

Hardie, J., and M. P. Osborne (1977) The electrical and mechanical properties of supercontracting body-wall muscles of the blowfly larva, Calliphora erythrocephala (MEIG). Comp. Biochem. Physiol. 57 A: $59-66$.

Hepler, J. R., P. Petrusz, and A. Rustioni (1986) Antisera to GABA, glutamate and aspartate: Characterization by immunoabsorption and immunocytochemistry. J. Histochem. Cytochem. 34: 110.

Hepler, J. R., C. Toomim, K. D. McCarthy, F. Conti, G. Battaglia, A. Rustioni, and P. Petrusz (1988) Characterization of antisera to glutamate and aspartate. J. Histochem. Cytochem. 36: 13-22.

Hertweck, H. (1931) Anatomie und Variabilität des Nervensystems und der Sinnesorgane von Drosophila melanogaster (Miegen). Z. Wiss. Zool. 139: 559-663.

Irving, S. N., and T. A. Miller (1980a) Ionic differences in "fast" and "slow" neuromuscular transmission in bodywall muscles of $\mathrm{Musca}$ domestica larvae. J. Comp. Physiol. 135: 291-298.

Irving, S. N., and T. A. Miller (1980b) Aspartate and glutamate as possible transmitters at the "slow" and "fast" neuromuscular junctions of the bodywall muscles of Musca larvae. J. Comp. Physiol. 135: 299-314.

Jan, L. Y., and Y. N. Jan (1976a) Properties of the larval neuromuscular junction in Drosophila melanogaster. J. Physiol. (Lond.) 262. 189-214.

Jan, L. Y., and Y. N. Jan (1976b) L-Glutamate as excitatory trans- 
mitter at the Drosophila larval neuromuscular junction. J. Physiol. (Lond.) 262: 215-236.

Jan, L. Y., and Y. N. Jan (1982) Antibodies to horseradish peroxidase as specific neuronal markers in Drosophila and grasshopper embryos. Proc. Natl. Acad. Sci. USA 72: 2700-2704.

Johansen, J., M. E. Halpern, K. M. Johansen, and H. Keshishian (1987) Bodywall muscle fibers of Drosophila larvae are innervated by both glutamate and aspartate immunoreactive nerve terminals. Soc. Neurosci. Abstr. 13: 1255.

Johansen, J., M. E. Halpern, and H. Keshishian (1988) Development of muscle innervation in Drosophila embryos and larvae. Soc. Neurosci. Abstr. 14: 451.

Osborne, M. P. (1967) The fine structure of neuromuscular junctions in the segmental muscles of the blowfly larva. J. Insect. Physiol. 13: $827-833$.

Robinson, N. L. (1981) Glutamate as the transmitter at fast and slow neuromuscular junctions of larval diptera. J. Comp. Physiol. 144: 139-143.

Toomim, C., K. McCarthy, and P. Petrusz (1986) Characterization of antibodies to glutamate and aspartate by immunoblot analysis and immunohistochemistry. Soc. Neurosci. Abstr. 12: 419.

Truman, J. W., and M. Bate (1988) Spatial and temporal patterns of neurogenesis in the central nervous system of Drosophila melanogaster. Dev. Biol. 125: 145-157.

Worden, M. K., J. L. Witten, and M. O'Shea (1985) Proctolin is a cotransmitter for the SETi motoneuron. Soc. Neurosci. Abstr. 11: 327.

$\mathrm{Wu}$, C.-F., and F. N. Haugland (1985) Voltage clamp analysis of membrane currents in larval muscle fibers of Drosophila: Alteration of polassiunn currents in Shaker mutants. J. Neurosci. 5: 2626-2640. Yoshikami, D., and L. M. Okun (1984) Staining of living presynaptic nerve terminals with selective fluorescent dyes. Nature 310: 53-56. 